\title{
In vitro Assessment and Characterization of the Growth and Life Cycle of Leishmania tropica
}

\author{
Qaisar Jamal*, Akram Shah, Syed Basit Rasheed and Muhammad Adnan \\ Department of Zoology, University of Peshawar, Peshawar, Pakistan
}

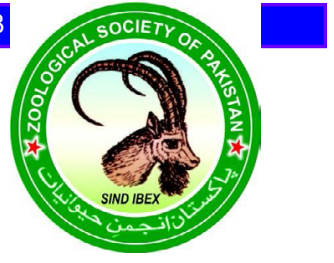

\begin{abstract}
A B S T R A C T
Present study aimed at determining the in vitro life cycle of Leishmania tropica. Temporal variability in the morphology and various morphotypes of the promastigotes were noted. Acquisition and propagation of axenic amastigotes was assessed. During promastigote culture, $\log$, mid-log, and late-log phases were observed respectively on day 4,5 and 6 . The stationary phase was observed on day 7. The day following inoculation, most of the promastigotes were nectomonads having long and slender bodies with roughly uniform width from anterior to near posterior end, having as long flagella as one and a half to twice the body. The nectomonads changed to leptomonads with a ratio of $5 \%$ and $44 \%$ on day 2 and 3 respectively. The body to flagellum ratio decreased in leptomonads giving a wider and shorter appearance than the nectomonads. On day 4, the log phase, the metacyclic promastigotes appeared in the culture. The metacyclic to leptomonad to nectomonad ratio was $27 \%, 43 \%$ and $29 \%$ respectively. The ratio of metacyclics steadily increased towards the mid-log $(53 \%)$, late-log $(67 \%)$, and stationary phase (83\%). Metacyclics had long flagella and short stumpy cell bodies with anterior end much broader than the posterior as compared to that in leptomonads and nectomonads. The posterior end of the body was bearing a slender tail like extension . During logarithmic growth, $17 \%$ of promastigotes were found dividing. During a 10 days transformation of axenic amastigotes, the day following inoculation to the day 3 different morphological forms were observed. The day 7 onward only rounded no flagellum ( $R N F=41$ $47 \%)$ and oval no flagellum $(\mathrm{ONF}=53-59 \%)$ forms were present. Viability remained $96-98 \%$ during transformation. Bulk growth of the promastigotes for prolong (over a week) duration resulted in the acidification of medium and change to viable amastigotes that could be successfully retransformed to promastigotes. Use of orthophosphoric acid for acquiring acidic medium to transform promastigotes to amastigotes proved effective.
\end{abstract}

\begin{tabular}{l} 
Article Information \\
\hline Received 18 July 2018 \\
Revised 11 May 2019 \\
Accepted 03 June 2019 \\
Available online 16 January 2020 \\
Authors' Contribution \\
QJ planned the work, did the \\
experimental lab work and wrote the \\
primary manuscript. AS supervised \\
the work. SBR helped in data analysis, \\
MA helped in prepration of the \\
manuscript. \\
Key words \\
Leishmania, Promastigotes, \\
Axenic amastigotes, Morphology, \\
Characterization
\end{tabular}

\section{INTRODUCTION}

L eishmaniasis, widely distributed in tropics and neotropics, is a vector-borne protozoal disease that ranks $9^{\text {th }}$ among the infectious diseases regarding global disease burden (Reithinger et al., 2007; Berry and BerrangFord, 2016). Human and mammalian leishmaniasis is characterized by a myriad of clinical spectra ranging from localized and ulcerative skin lesions (cutaneous leishmaniasis) to non-ulcerative nodules (diffused cutaneous leishmaniasis) to the inflammation of mucus membranes (muco-cutaneous leishmaniasis) to infection of visceral organs (visceral leishmaniasis; the fatal form of the disease causing maximum deaths). Execept in some cases, where one species can infect different foci, the different forms are caused by different species (Reithinger et al., 2007). It is currently reported from 98 countries around the globe with an overall global prevalence of 12 million cases, annual death incidence of 70,000 and 350 million

\footnotetext{
* Corresponding author: qaisar.jamal21@uop.edu.pk 0030-9923/2020/0002-0447 \$ 9.00/0

Copyright 2020 Zoological Society of Pakistan
}

people at risk. The cutaneous and visceral leishmaniases have an annual global incidence of 0.7-1.2 million and 0.2-0.4 million respectively. In Pakistan it is estimated that approimately 21-35 thousand cases of CL occur annually (Jamal et al., 2015; Reithinger et al., 2007; Rodrigues et al., 2015; Rock et al., 2015). Most of the VL cases (90\%) are reported from India, Brazil, Sudan, Bangladesh, Ethiopia and southern Sudan having annual death toll of 20-40 thousands (Gradoni, 2013; Kaur et al., 2015). Colombia, Peru, Brazil, Costa Rica, Algeria, North Sudan, Ethiopia, Afghanistan, Iran, and Syria contribute $70-75 \%$ of the 10 million cases of the cutaneous leishmaniasis (Ambit et al., 2011; Alvar et al., 2012).

Leishmania, the etiologic agent of leishmaniasis, possesses a heteroxenous life cycle, swinging between flagellated promastigote and aflagellated amastigote form occurring respectively in invertebrate (sandfly gut) and vertebrate (a variety of lizards and mammals including human) macrophages. Both the forms of the parasite can be maintained in axenic cultures using defined media (CysneFinkelstein et al., 1998; Sacks, 1989; Sacks et al., 2000). The promastigote form has been demonstrated to show morphologically and functionally different morphotypes 
both in vivo (Killick-Kendrick, 1979; Sacks and Perkins, 1985) and in vitro (Sacks et al., 1995; Lira et al., 1998). As compared to in vivo existence of morphotypes (Schlein et al., 1993; Killick-Kendrick et al., 1996; Gossage et al., 2003; Oliveira et al., 2009; Ramalho-Ortigao et al., 2010; Dostálova and volf, 2012), in vitro existence is less established. Limited number of studies, as stated before, with few species of Leishmania, have investigated these morphological forms in axenic development. Evaluation of such variability is really important for infection kinetics of the parasite and for their conversion to axenic amastigotes. Amongst the variety of different morphotypes, metacyclic form is capable of causing infection both in vivo and in vitro. In axenic culture, the metacyclogenesis reaches at different rates for different species. The published record for $L$. tropica metacyclogenesis in axenic growth is scarce. This study aimed at elucidation of metacyclogenses of $L$. tropica promastigotes and acquisition of axenic amastigotes.

\section{MATERIALS AND METHODS}

\section{Growth of promastigotes}

Promastigotes were cultured in RPMI-1640 medium (Sigma Life Sciences) at a density of $1 \times 10^{6} / \mathrm{mL}$ in $25 \mathrm{~mL}$ nonvented culture flask. The medium was supplemented with $10 \%$ heat inactivated fetal calf serum (hiFCS, Sigma Life Sciences), $100 \mu \mathrm{g} / \mathrm{mL}$ streptomycin (Sigma Life Science), 100 IU penicillin (Sigma Life Sciences) and L-glutamine. The growth was monitored daily by counting in neubauer haemocytometer over a week.

\section{Evaluation of morphological variations of promastigotes during culture}

Morphologic variability was monitored daily through Giemsa stained smear over a period of one week. Usually a drop of culture was smeared on an albuminized slide and fixed in methanol after air drying at room temperature. Various trial concentrations of the aqueous methanol were tried to fix the parasite cells. Fixed smears were stained with Giemsa stain. Smears were observed under the oil immersion objective and photographed. Temporal variation in the length, width, and shape of the cell and length of the flagellum were qualitatively assessed to determine different promastigote morphotypes in the axenic culture. Metacyclics were also revealed through scanning electron microscopy.

Cultivation of axenic amastigotes and evaluation of morphology

Axenically grown amastigotes have become a great deal of interest for drug screening and biochemical as well as molecular variability between the promastigote and amastigote stages. A variety of different protocols have been followed to axenically transform promastigotes to amastigotes for different species. In the present study, $1 \times 10^{6} / \mathrm{mL}$ promastigotes in the stationary phase were grown at $37^{\circ} \mathrm{C}$ in humidified conditions lacking $\mathrm{CO}_{2}$ in RPMI 1640 medium fortified with $20 \%$ heat inactivated fetal calf serum (hiFCS: Sigma Lifescience), $100 \mu \mathrm{g} / \mathrm{mL}$ streptomycin, $100 \mathrm{IU}$ penicillin and L-glutamine. The $\mathrm{pH}$ of the medium was adjusted at 4.4 using phosphoric acid ( $85 \%$ aqueous solution BDH Chemicals). Giemsa staining, and phase contrast microscopy was used to monitor the transformation on daily bases while fully transformed amastigotes were also visualized through scanning electron microscope.

During the promastigote to amastigote transformation, the change in cell shape was observed daily by Giemsa smearing and expressed in terms of percentage from elongated promastigote form in the initial inoculum to the fully round form with a variety of oval forms in between. Presence and absence and length of the flagellum was also noted. Reduction in the cell size was determined through micrometry. The size measurement was mostly based on the fully round and oval individuals. The size was expressed as mean of 20 randomly selected individual cell measurements.

\section{Acquisition of axenic amastigotes by starvation}

Amastigotes were acquired in the in vitro culture when the culture was kept starved without medium change, accidentally for the first time, and then several times when repeated intentionally.

\section{Viability during transformation}

Viability of cells is a necessary aspect in the drug sensitivity and biological studies. The viability of leishmanial cells during promastigote to amastigote transformation was checked daily over a week using trypan blue exclusion and was expressed as percentage of viable cells. After complete transformation, the amastigotes were culture in the normal RPMI-1640 as mentioned above for promastigotes to check the viability.

\section{RESULTS}

\section{Growth curve of $\mathrm{L}$. tropica $K W H 23$}

The growth of L. tropica KWH23 was observed by growth curve over one week period of the culture (Fig. 1). For the first three days of the culture, a stunted growth was seen. On day 4 , the culture reached log phase of the growth. On day 5 to 6 the mid and late $\log$ phases of $L$. tropica promastigotes development were achieved. On 
day 7 , the promastigote culture entered the stationary phase $\left(1 \times 10^{7} / \mathrm{mL}\right)$. In stationary phase, the promastigotes became sluggish and were slithering around. Cells were looking stumpy with long flagella. The body looked like a carrot with bulging anterior and tapering posterior ends.

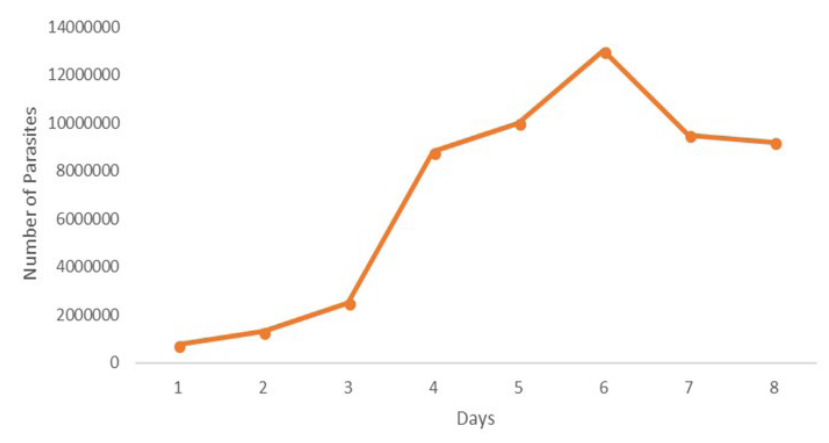

Fig. 1. Growth curve of Leishmania tropica KWH23 over a week.

\section{Revision of strain nomenclature}

Leishmania tropica KWH23 strain was originally isolated from a 5-year-old boy belonging to Jamrud (an endemic focus for cutaneous leishmaniasis) area of Kyber Agency, then known as Federally Administered Tribal Area (FATA) Pakistan in 2010 visiting Kuwait Teaching Hospital, Peshawar, Khyber Pakhtunkhwa Pakistan for intra lesional treatment. It was isolated by Nazma Habib Khan during her Ph D from London School of Hygiene and Tropical Medicine, London UK. Since then it has been labelled with the name where KWH comes from the name of the hospital where the strain originated and 23 refers to the serial number of the patient studied. According to the strain nomenclature for Leishmania isolates (leishnet.net), it must contain four parts separated by a slash (/). The first part is a reference to the host from which it is isolated, usually a four-letter abbreviation; first of which represents the class of the host in the hierarchic classification followed by the first three letters of the genus of the host. The second part is a two-letter abbreviation of the country of origin, a list of which is available online. The third part is the year of isolation and the fourth part is not fixed and is on the choice of the person who has done the isolation, which might be the original patient ID in the hospital record or of the researcher, thus revising this strain to $\mathrm{MHOM} /$ PK/2010/KWH23.

\section{Axenic promastigote morphotypes}

Various morphotypes were determined through one week development. Culture was initiated from stationary phase promastigotes. It was infinite passage number of the strain KWH23. On day $1^{\text {st }}$ almost all of the promastigotes were nectomonads (Fig. 2B) having long, slender and progressively tapering bodies with long flagella about one and a half to twice as long as the body. Next day only $5 \%$ of the promastigotes were leptomonads (Fig. 2C) with still slender and long cells and longer flagella almost indistinguishable from those of nectomonads. In leptomonads, the cell body becomes somewhat less tapering posteriorly and cell to flagellum ratio decreses. On day third, the ratio of leptomonads rose to $44 \%$. During the $\log$ phase on day 4 , metacyclics appeared in the culture. The matacyclics to leptomonads to nectomonads were $27 \%, 43 \%$ and $29 \%$ respectively. Metacyclics had long flagella and short stumpy cell bodies with anterior end much broader than the posterior as compared to that in leptomonads and nectomonads. The posterior end of the body was bearing a slender tail like extension (Fig. 2 D, E, F). Cell number got increased till day 6 ( late log phase) where onward the stationary phase reached and the number of metacyclics increased progressively. Microscopic assessment of the in vitro development of L. tropica in the present study represented few procyclic (Fig. 2A).

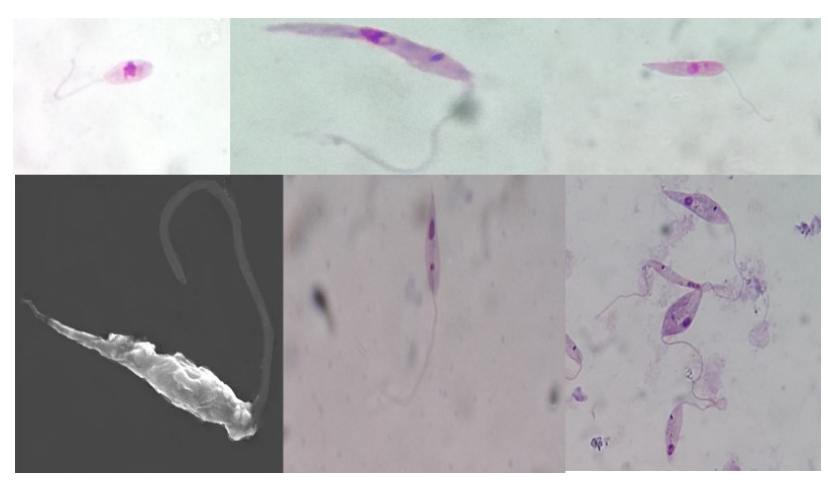

Fig. 2. Different stages of $L$. Tropica KWH23 promastigotes in culture (See text for description).

Division of L. tropica promastiogtes in axenic development During logarithmic growth, $17 \%$ of promastigotes were found dividing, so features of the dividing cells were observed (Fig. 3). Most of the individuals duplicated the flagellum first following division of the kinetoplast and the nucleus respectively. However, in rare cases, division of the kinetoplast and nucleus preceded flagellar splitting (Fig. 3C).

\section{Cultivation of axenic amastigotes}

After 24 hours of incubation under the abovementioned conditions, the metacyclic promastigotes started to change in the overall morphology. As per previously described criteria of transformation from promastigote to amastigote, major changes have been observed regarding 
Table I. Promastigote to amastigote transformation ratio over 10-day span based on cell morphology.

\begin{tabular}{lllllllllllll}
\hline $\begin{array}{l}\text { \% Shape } \rightarrow \\
\text { Day } \downarrow\end{array}$ & RNF & RBVF & RSF & RLF & ONF & OBVF & OSF & OLF & ENF & EBVF & ESF & ELF \\
\hline 1. & 03 & 05 & 05 & 02 & 04 & 25 & 27 & 09 & 08 & 05 & 04 & 03 \\
2. & 10 & 09 & 5 & 01 & 23 & 27 & 20 & 02 & 0 & 0 & 02 & 01 \\
3. & 09 & 08 & 07 & 01 & 31 & 40 & 03 & 01 & 0 & 0 & 0 & 0 \\
4. & 19 & 13 & 0 & 0 & 68 & 0 & 0 & 0 & 0 & 0 & 0 & 0 \\
5. & 31 & 06 & 0 & 0 & 63 & 0 & 0 & 0 & 0 & 0 & 0 & 0 \\
6. & 29 & 09 & 0 & 0 & 62 & 0 & 0 & 0 & 0 & 0 & 0 & 0 \\
7. & 41 & 0 & 0 & 0 & 59 & 0 & 0 & 0 & 0 & 0 & 0 & 0 \\
8. & 44 & 0 & 0 & 0 & 56 & 0 & 0 & 0 & 0 & 0 & 0 & 0 \\
9. & 47 & 0 & 0 & 0 & 53 & 0 & 0 & 0 & 0 & 0 & 0 & 0 \\
10. & 43 & 0 & 0 & 0 & 57 & 0 & 0 & 0 & 0 & 0 & 0 & 0 \\
\hline
\end{tabular}

RNF, rounded no flagellum; RBVF, rounded barely visible flagellum; RSF, rounded stumpy flagellum; RLF, rounded long flagellum; ONF, oval no flagellum; OBVF, oval barely visible flagellum; OSF, oval stumpy flagellum; OLF, oval long flagellum; ENF, elongated no flagellum; EBVF, elongated barely visible flagellum; ESF, elongated stumpy flagellum; ELF, elongated long flagellum.

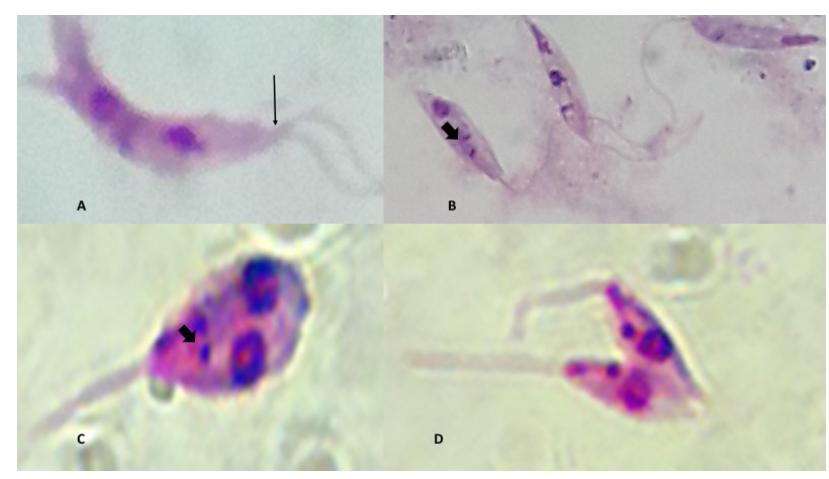

Fig. 3. Division of the L. tropica promastigotes in axenic development. Cell with 2 flagella single kinetoplast and single nucleus (A), Cells with single flagellum, two kinetoplasts and single nucleus (B), Cell with Single flagellum, two kinetoplasts and two nuclei $(\mathrm{C})$ and Cells with two flagella, two kinetoplasts and two nuclei near to the completion of cytokinesis (D). Solid arrows show kinetoplasts and the others indicate flagella.

the morphological changes in cell shape and length of flagellum (Fig. 4).

\section{Shape and flagellar changes during transformation}

The changes in shape of the cell and length of the flagellum during promastigote to amastigote transformation has been noted (Table I). The results were presented as percentage per 100 cells counted in a Giemsa stained smear. In a 10 days daily monitoring of the transformation process several different morphotypes were observed. There was a gradual loss of the flagellum with individual variability. As is clear from the table on day 1; the following day of inoculation, all the different morphotypes were seen. Up till day 3 a mixture of the morphotypes were present. Day 4 and onward only the rounded no flagellum and oval no flagellum along with few percent of rounded barely visible flagellum forms were present in the culture. Although the transformation process started right on the following day of inoculation, but proper amastigote forms were transformed after day 4 and onward. The fully transformed culture contained the rounded and oval forms. However, oval cells predominated the rounded ones throughout the culture. Our recent observations of amastigotes of $L$. tropica isolated from CL lesions from the type locality of the strain showed both oval and round forms (Fig. 4).

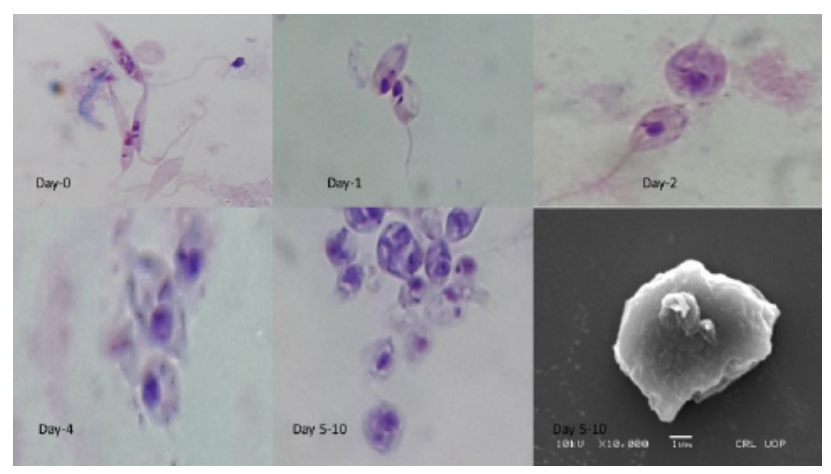

Fig. 4. Promastigote to amastigote transformation. Change in the cell morphology and loss of flagellum is seen progressively from day $0-10$. Lesion amastigotes are indicated by the arrow in the last inset.

Cell size as well as length of flagellum experienced tremendous reduction during the transformation of 
amastigotes. This reduction in cell size was abrupt. There was a maximum reduction in cell length on day first and second after which the size of the amastigote form became rather more constant (Table II). Fully transformed amastigote form varied from little oval or near round to fully rounded (Table III).

Table II. Transformation of promastigotes to amastigotes in terms cell size reduction over a week.

\begin{tabular}{lcllll}
\hline Days & \multicolumn{1}{c}{ Mean } & Variance & Stdev. & CI Var. & CI Stdev. \\
\hline 0 & 10.96667 & 2.363529 & 1.537377 & $1.4-5.4$ & $1.2-2.1$ \\
1 & 4.511111 & 0.704575 & 0.83939 & $0.4-1.7$ & $1.18-1.45$ \\
2 & 3.927778 & 0.296242 & 0.544281 & $0.17-0.7$ & $0.413-0.84$ \\
3 & 3.277778 & 0.065359 & 0.255655 & $0.036-0.15$ & $0.189-0.387$ \\
4 & 3.111111 & 0.045752 & 0.213896 & $0.025-0.1$ & $0.158-0.316$ \\
5 & 3.194444 & 0.062908 & 0.250816 & $0.035-0.138$ & $0.187-0.371$ \\
6 & 3.111111 & 0.045752 & 0.213896 & $0.025-0.1$ & $0.158-0.316$ \\
7 & 3.138889 & 0.053105 & 0.230444 & $0.030-0.118$ & $0.173-0.343$ \\
\hline
\end{tabular}

Table III. Size of the fully round and oval form of amastigote.

\begin{tabular}{llllll}
\hline \multicolumn{5}{c}{ Fully Round Form } & \\
\cline { 2 - 6 } & Mean & Variance & Stdev. & CI var, & CI Stdev \\
\hline Day-5 & 3.18 & 0.101714 & 0.318927 & $0.05-0.25$ & $0.224-0.5$ \\
Day-10 & 3.146667 & 0.095524 & 0.309069 & $0.048-0.225$ & $0.219-0.474$ \\
& Oval form & & & & \\
Length & 3.653333 & 0.132667 & 0.364234 & $0.071-0.33$ & $0.266-0.574$ \\
Width & 2.94 & 0.084 & 0.289828 & $0.045-0.21$ & $0.212-0.458$ \\
\hline
\end{tabular}

\section{Viability during transformation}

Viability of cells is a necessary aspect in the drug sensitivity and biological studies. The viability of leishmanial cells during promastigote to amastigote transformation was checked daily during the study period using trypan blue exclusion and was expressed as percentage of viable cells. It varied from $96-98 \%$.

\section{Natural acquisition of axenic amastigotes}

During the study period, when several times the culture was kept starved for a week to ten days at $26^{\circ} \mathrm{C}$ and absence of $\mathrm{CO}_{2}$ the promastigotes changed to amastigotes due to change in $\mathrm{pH}$ of medium although this occurrence was purely accidental for the first time. Colour of the medium changed from light pink to light yellow with final $\mathrm{pH}$ of 4.8-5. The culture medium (having 10 $\%$ FCS) started to change its colour after day 3 with $\mathrm{pH}$ falling to 6.8 having only promastigotes. On day $5^{\text {th }}$ the
$\mathrm{pH}$ fell to 6 but it still was having only promastigotes in late log phase. The $\mathrm{pH}$ fell gradually to 5.5 on day 7 with $82 \%$ promastigotes and the rest were partially changed amastigotes having rounded to oval or stumpy body but long flagellum. On day 9 the $\mathrm{pH}$ came to about 5 and the percentage of amastigotes increased to $80 \%$ mostly having rounded body and stumpy to rudimentary flagellum. On day 10 and onward the $\mathrm{pH}$ came to 4.8 and the ratio of amastigotes rose to $91 \%$. Viability of the amastigotes was $97 \%$ as evaluated by trypan blue exclusion. The culture had $1.2 \times 10^{7}$ amastigotes per $\mathrm{mL}$. When grown in medium for promastigotes such amastigotes successfully transformed back to promastigotes. Natural transformation was slower than the one performed in the defined medium for axenic amastigotes. However, the results were satisfactory, and the yield was enough. Such transformation yielded enough axenic amastigotes for one-time use. The amastigotes could be successfully propagated through sub-passaging in the defined medium.

Some requirements for counting, fixation and smearing of Leishmania tropica $\mathrm{KWH} 23$

Counting is a necessary process in culturing and propagation as well as in vitro and in vivo anti-leishmanial assays of compounds. Inactivation of the L. tropica promastigote is needed for counting in haemocytometer as they are actively motile. Amastigotes are, however, stationary and need no such inactivation. During the present study, 10-25\% ethanol was found far beyond satisfactory to inactivate them to be easily studied under microscope in haemocytometer. Ten percent aqueous methanol solution for 10-30 seconds gave excellent results in fixing both the promastigote and amastigote forms of the parasite. To attach the parasite to the slide surface, egg albumin was used. Fresh albumin was less efficient in adhesion of the parasite to slide but a day to a week older albumin was found very much effective in attaching the cells to the slide surface. Amastigotes were found less efficient in sticking to the surface of albumenized slide than the promastigotes. A $2 \% \mathrm{~V} / \mathrm{V}$ working Giemsa stain for 10-20 minutes at room temperature gave excellent results in staining both the promastigotes and amastigotes. Such a working Giemsa stain worked satisfactorily for 2 weeks. Fresh stain replacement after two weeks is, however, recommended.

\section{DISCUSSION}

Morphological variability of promastigote stage in different species of Leishmania have been described in the vector host. Several different morphotypes occurs during development in sand fly. Each of these morphotypes also presents bichemical variability and varied virulence to 
the host (Schlein, 1993; Ismael et al., 1998; Gossage et al., 2003; Kamhavi, 2006; Ramalho-Ortigao et al., 2010; Oliveira et al., 2009; Dougall et al., 2011). The description of such morphotypes in axenic development is apparently difficult, partly because of temporal association of such developmental form and partly due to great deal of speciesspecific variations. For these reasons, limited number of studies have been attempted to assess the morphological forms in the axenic cultivation of the promastigotes in few Leishmania species, L. donovani, L. mexicana and L. major being the prominent ones (da Silva and Sacks, 1987; Sacks, 1989). However, axenic growth of the promastigotes reflect several different morphotypes seen in the in vivo development in the vector host. Different morphotypes reported in the sand fly vector can also be detected in the in vitro culture (Sacks et al., 1984; Gossage et al., 2003; Lei et al., 2010a)

Initiated from lesion amastigotes, Bates (1994) demonstrated, various morphotypes of L. mexicana axencally. The initial amastigote to promastigote transformation was achieved in 2 days. Logarithmic growth, with a mix of different morphotypes in the multiplicative stage, was noted to occur on days 3 and 4. At this stage (3-4 days), few infective metacyclic promastigotes were observed. Most of the promastigotes on the late day 3 were longest $(15.22 \pm 3.49 \mu \mathrm{M})$ and extremely slender than noted on any other day and were carrying long flagella. However, flagellar and body length were comparable. These were morphologically very much similar to the nectomonads noted in in vivo studies. On day $7^{\text {th }}$, the promastigotes reached to the stationary phase and metacyclogenesis was on the peak. In 9 days old axenic culture, however, the density of metayclics reached to the $95 \%$. The metacyclic promastigotes became stumpy, broader anteriorly, needle sharp posteriorly and were with long flagella. The flagella were 1-2 times longer than the cell size $(7.59 \pm 1.15 \mu \mathrm{M})$. These findings are in a good consensus with what we have come across in this study. Short and slender metacyclic promastigotes with long flagella have been demonstrated in stationary cultures by other investigators also (Sacks and Perkinn, 1984; Grimm et al., 1991).

The ratio of metacyclics to other forms is in inverse relationship to the number of passages. It usually decreases in long-term axenic cultivation. Fresh cultures that have gone through few passages get fast metacyclogenesis with higher densities of metacyclics than the older cultures (Cysne-Finkelstein et al., 1998). Results of the present study are strongly supported by the in vivo results of Dougall et al. (2011). Their study have reported similar kind of morphological forms of promastigote of a yet unidentified species of Leishmania isolated from day biting midges of genus Forcipomyia. They found very long slender nectomonads with long flagella. The leptomonads they isolated were rather short banana like with relatively short flagella. Their metacyclics, however, varied from needle long with long flagella to anteriorly broad and posteriorly needle sharp having long flagella. Lei et al. (2010) have reported the existence of procyclic, nectomonad, leptomonad and metacyclics in culture. They have shown very similar body lenghts for procyclics, leptomonads and metacyclics $(6-11 \mu \mathrm{M})$. However, nectomanads were found more than $12 \mu \mathrm{M}$ in length. All of the stages they noted have been of very similar morphology to what we have found in the present study.

Lectins obtained from several different plants including peanut, is used to purify metacyclic promastigotes from culture. This compound agglutinates the procyclic and other forms leaving metacyclics. Metacyclics and non-metacyclics separated from stationary culture of $L$. amazonensis have been reported to be morphologically different. The non-metacyclics shown so have been long and almost equally broad along their length except near the posterior tip. These have been shown to have very long flagella. These are characteristics of the nectomonads. The mectacyclics have been short and stumpy with broader anterior half and much narrower posterior half. These have been shown to possess very long flagella (Saravia et al., 2005). This work support our results in the present study. Soares et al. (2005) also noted similar kind of results for L. braziliensis. The procyclic form was found to be very much variable in size of the cell a flagellum. They probably considered all the non-metacyclic forms as procyclic that varied in cell size from rounded to elongated individuals. However, the metacyclics were defined as smaller and thinner,usually posteriorly, bodies with long flagella.

When grown under different conditions of temperature and $\mathrm{pH}$, the metacyclic promastigotes of $L$. mexicana showed different growth and pro-amastigote transformation kinetics. At a temperature of $26^{\circ} \mathrm{C}$ and $\mathrm{pH}$ of 7.2 no transformation occurred. With these conditions, however, in some experiments transitional forms have been seen for short duration. At $26^{\circ} \mathrm{C}$ and $\mathrm{pH} 5.5$, greater degree of transformation have been observed but it was followed by reversion to promastigotes in 24 hours. When the transformation was checked at $32^{\circ} \mathrm{C}$ and $\mathrm{pH}$ 7.2 , within 12 hours almost all of the cells entered to the intermediate forms that sustained for 48 hours. Although the intermediate forms did not revert to the promastigote stage but also did not fully transformed to amastigote stage. A temperature and $\mathrm{pH}$ panel of $32^{\circ} \mathrm{C}$ and 5.5 was the most effective in bringing the desired transformation over 48-96 hours (Bee et al., 2001). These conditions for transformation are in close coincidence of our observation. 
Similarly, the observation of various transitional stages in the mentioned study is shared with what we noted in this study. However, we did not see any reversion like the mentioned workers at the ideal combination they used. A difference from what they have noted that we achieved quite good amastigote transformation at $26^{\circ} \mathrm{C}$ and $4.8 \mathrm{pH}$ in the natural transformation.

Metacyclic promastigotes of $L$. mexicana grown in Schnider's medium suppliemented with $20 \%$ fetal calf serum at $25^{\circ} \mathrm{C}$ and $5.5 \mathrm{pH}$ have been successfully transformed to amastigotes by rising simply the temperature to $32^{\circ} \mathrm{C}$ in the parent culture. After 24 hours of the changed conditions of the temperature, more than $80 \%$ of the cells changed to rounded amastigotes with complete loss of flagellum. The remaining were the intermediate forms differing in the cell and flagellar morphology. By the day 2, however, $100 \%$ transformation was seen (Bates, 1994). These findings present a strong correlation of temperature and $\mathrm{pH}$ to the transformation process. Species-specific variability can, however, not be ruled out. Generally, a temperature and $\mathrm{pH}$ combination of $32-37^{\circ} \mathrm{C}$ and $4.5-5.5$ seems feasible for bringing satisfactory transformation. Entirely transformed amastigotes may be ovoid to fully rounded in almost all species of Leishmania.

\section{CONCLUSION}

Morphological variability in the in vitro development of L. tropica can be determined microscopically. The present study will provide guidelines for the subsequent workers in acquisition of any specific morphotype for their use. This will also help them in acquiring axenic amastigotes of the species. A comprehensive protocol for processing the promastigotes as well as amastigotes for both light and electron microscopy will be of help to the coming workers.

\section{ACKNOWLEDGEMENTS}

The authors are extremely thankful to Professor Nasim Siddiqui Memorial Parasitology Laboratory and Leishmania Molecular and Culture Lab of the Department of Zoology, University of Peshawar for providing material support for this study.

\section{Statement of conflict of interset}

The authors hereby declare no conflict of interest.

\section{REFERENCES}

Alvar, J., Velez, I.D., Bern, C., Herrero, M., Desjeux, P., Cano, J., Jannin, J. and den Boer, M., 2012.
Worldwide and global estimates of its incidence. PLoS One, 7: e35671. https://doi.org/10.1371/ journal.pone.0035671

Ambit, A., Woods, K.L., Cull, B., Coombs, G.H. and Mottram, J.C., 2011. Morphological events during the cell cycle of Leishmania major. Eukary. Cell, 10: 1429-1438. https://doi.org/10.1128/EC.0511811

Bates, P.A., 1994. Complete developmental cycle of Leishmania mexicana in axenic culture. Parasitology, 108: 1-9.

Bee, A., Culley, F.J., Alkhalife, I.S., BodmanSmith, K.B., Raynes, J.G. and Bates, P.A., 2001. Transformation of Leishmania mexicana metacyclic promastigotes to amastigote-like forms mediated by binding of human C-reactive protein. Parasitology, 122: 521-529. https://doi. org/10.1017/S0031182001007612

Berry, I. and Berrang-Ford, L., 2016. Leishmaniasis, conflict, and political terror: A spatio-temporal analysis. Soc. Sci. Med., 167: 140-149. https://doi. org/10.1016/j.socscimed.2016.04.038

Cysne-Finkelstein, L., Temporal, R.M., Alves, F.A. and Leon, L.L., 1998. Leishmania amazonensis: Long-term cultivation of axenic amastigotes is associated to metacyclogenesis of promastigotes. Exp. Parasitol., 89: 58-62. https://doi.org/10.1006/ expr.1998.4276

Da Silva, R.P. and Sacks, D.L., 1987. Metacyclogenesis is a major determinant of Leishmania promastigote virulence and attenuation. Infect. Immun., 55: 2802-2807.

Dostálová, A. and Volf, P., 2012. Leishmania development in sand flies: parasite-vector interactions overview. Parasit. Vectors, 5: Art. No. 276. https://doi.org/10.1186/1756-3305-5-276

Dougall, A.M., Alexander, B., Holt, D.C., Harris, T., Sultan, A.H., Bates, P.A., Rose, K. and Walton, S.F., 2011. Evidence incriminating midges (Diptera: Ceratopogonidae) as potential vectors of Leishmania in Australia. Int. J. Parasitol., 41: 571579. https://doi.org/10.1016/j.ijpara.2010.12.008

Gossage, S.M., Rogers, M.E. and Bates, P.A., 2003. Two separate growth phases during the development of Leishmania in sand flies: implications for understanding the life cycle. Int. J. Parasitol., 33: 1027-1034. https://doi.org/10.1016/S00207519(03)00142-5

Gradoni, L., 2013. Epidemiological surveillance of leishmaniasis in the EuropeanUnion:operationaland research challenges. Euro. Surveill., 18: pii=20539. Available online: http://www.eurosurveillance. 
org/ViewArticle.aspx?ArticleId=20539 https://doi. org/10.2807/1560-7917.ES2013.18.30.20539

Grimm, F., Brun, R. and Jenni, L., 1991. Promastigote infectivity in Leishmania infantum. Parasitol. Res., 77: 185-191. https://doi.org/10.1007/BF00930856

Ismaeel, A.Y., Garmson, J.G., Molyneux, D.H. and Bates, P.A., 1998. Transformation, development, and transmission of axenically cultured amastigotes of Leishmania mexicana in vitro and in Lutzomyia longipalpis. Am. J. Trop. Med. Hyg., 59: 421-425. https://doi.org/10.4269/ajtmh.1998.59.421

Jamal, Q., Khan, N.H., Wahid, S., Awan, M.M., Sutherland, C. and Shah, A., 2015. In-vitro sensitivity of Pakistani Leishmania tropica field isolate against buparvaquone in comparison to standard antileishmanial drugs. Exp. Parasitol., 154: 93-97. https://doi.org/10.1016/j.exppara.2015.04.017

Kamhawi, S., 2006. Phlebotomine sand flies and Leishmania parasites: friends or foes? Trends Parasitol., 22: 439-445. https://doi.org/10.1016/j. pt.2006.06.012

Kaur, H., Seifert, K., Hawkes, G.E., Coumbarides, G.S., Alvar, G. and Croft, S.L., 2015. Chemical and bioassay techniques to authenticate quality of the anti-leishmanial drug Miltefosine. Am. J. Trop. Med. Hyg., 92 (Suppl 6): 31-38. https://doi.org/10.4269/ ajtmh.14-0586

Killick-Kendrick, R., 1979. Biology of Leishmania in phlebotomine sandflies. In: Biology of Kinetoplastida (eds. W.H.R. Lumsden and D.A. Evans). vol. 2. Academic Press, London, pp. 395-460. https://doi. org/10.1051/parasite/1996031055

Killick-Kendrick, R., Killick-Kendrick, M., Tang, Y. and Bastien, P., 1996. Metacyclic promastigotes of Leishmania in the salivary glands of experimentally infected phlebotomine sandflies. Parasite, 3: 55-60.

Lei, S.M., Ramer-Tait, A.E., Dahlin-Laborde, R.R., Mullin, K. and Beetham, J.K., 2010. Reduced hamster usage and stress in propagating Leishmania chagasi promastigotes using cryopreservation and saphenous vein inoculation. J. Parasitol., 96: 103108. http://leishnet.net/site/?q=node/10; https://doi. org/10.1645/GE-2192.1

Lira, R., Méndez, S., Carrera, L., Jaffe, C., Neva, F., Sacks, D.L., 1998. Leishmania tropica: the identification and purification of metacyclic promastigotes and use in establishing mouse and hamster models of cutaneous and visceral disease. Exp. Parasitol., 89: 331-342. https://doi.org/10.1006/expr.1998.4283

Oliveira, F., Jochim, R.G., Valenzuela, J.G. and Kamhawi, S., 2009. Sand flies, Leishmania, and transcriptomeborne solutions. Parasitol. Int., 58: 1-5. https://doi. org/10.1016/j.parint.2008.07.004

Ramalho-Ortigao, M., Saraiva, E.M. and Traub-Csekö, Y.M., 2010. Sand fly-Leishmania interactions: long relationships are not necessarily easy. Open Parasitol. J., 4: 195-204. https://doi. org/10.2174/1874421401004010195

Reithinger, R., Dujardin, J., Louzir, H., Pirmez, C., Alexander, B. and Brooker, S., 2007. Cutaneous leishmaniasis. Lancet Infect. Dis., 7: 581-96. https:// doi.org/10.1016/S1473-3099(07)70209-8

Rock, K.S., le Rutte, E.A., de Vlas, S.J., Adams, E.R., Medley, G.F. and Hollingsworth, T.D., 2015. Uniting mathematics and biology for control of visceral leishmaniasis. Trends Parasitol., 31: 251. https:// doi.org/10.1016/j.pt.2015.03.007

Rodrigues, I.A., Mazotto, A.M., Cardoso, V., Alves, R.L., Amaral, A.C.F., Silva, J.R. deA., Pinheiro, A.S. and Vermelho, A.B., 2015. Natural products: Insights into leishmaniasis inflammatory response. Mediators Inflamm., Article ID 835910, 12 pages. https://doi.org/10.1155/2015/835910

Schlein, Y., 1993. Leishmania and sandflies: Interactions in the life cycle and transmission. Parasitol. Today, 9: 255-258. https://doi.org/10.1016/01694758(93)90070-V

Sacks, D.L. and Perkins, P.V., 1984. Identification of an infective stage of Leishmania promastigotes. Science, 223: 1417-1419. https://doi.org/10.1126/ science.6701528

Sacks, D.L. and Perkins, P.V., 1985. Development of infective stage of Leishmania promastigotes within phlebotomine sand flies. Am. J. Trop. Med. Hyg., 34: 456-459. https://doi.org/10.4269/ajtmh.1985.34.456

Sacks, D.L., 1989. Metacyclogenesis in Leishmania promastigotes. Exp. Parasitol., 69: 100-403. https:// doi.org/10.1016/0014-4894(89)90176-8

Sacks, D.L., Pimenta, P.F.P., McConville, M.J., Schneider, P. and Turco, S., 1995. Stage-specific binding of Leishmania donovani to the sand fly midgut is regulated by conformational changes in the abundant surface lipophosphoglycan. $J$. exp. Med., 181: 685-697. https://doi.org/10.1084/ jem.181.2.685

Sacks, D.L., Modi, G., Rowton, E., Späth, G., Epstein, L., Turcoi, S.J. and Beverley, S.M., 2000. The role of phosphoglycans in Leishmania-sand fly interactions. Proc. Nat. Acad. Sci. USA., 97: 406-411. https://doi. org/10.1073/pnas.97.1.406

Saraiva, M.E., Pinto-da-Silva, L., Wanderley, J.L.M., Bonomo, A.C., Barcinski, M.A. and Moreira, M.E.C., 2005. Flow cytometric assessment of Leishmania spp metacyclic differentiation: 
Validation by morphological features and specific markers. Exp. Parasitol., 110: 39-47. https://doi. org/10.1016/j.exppara.2005.01.004

Schlein, Y. 1993. Leishmania and sandflies: Interactions in the life cycle and transmission. Parasitol. Today, 9: 255-258. https://doi.org/10.1016/01694758(93)90070-V
Soares, R.P.P., Cardoso, T.L., Barron, T., Araújo, M.S.S., Pimenta, P.F.P. and Turco, S.J., 2005. Leishmania braziliensis: a novel mechanism in the lipophosphoglycan regulation during metacyclogenesis. Int. J. Parasitol., 35: 245-253. https://doi.org/10.1016/j.jpara.2004.12.008 